\title{
ASSESSMENT STRATEGY OF HUMAN UPPER FOREARM INTER-RELATION AND MUSCLE FATIGUE
}

\author{
W. M. B. WAN DAUD ${ }^{1}$, N. $\operatorname{ABAS}^{1}$ and M. O. TOKHI ${ }^{2}$ \\ ${ }^{1}$ Department of Automatic Control and Systems Engineering, \\ The University of Sheffield, United Kingdom \\ ${ }^{2}$ School of Engineering, London South Bank University, \\ United Kingdom.
}

\begin{abstract}
Surface electromyography (EMG) signals classification is currently applied in various prostheses and arm controls using various classification methods. The limited robustness in practical EMG control applications has become an important matter of research consideration. The precision of EMG signal features and parameters proportionally vary with muscle fatigue (MF). The major challenge for the study is to identify the MF manifestation in the EMG signal, so that the control performance is improved. This can be done by the improvement of data collection practicality, features extraction and classification. Hence, fundamental study is performed by investigating the signals acquired from the human upper forearm (UFA) to determine muscle characteristics and to establish the inter-relationship between both muscles of the forearm and upper arm. The aim of the present study is to investigate the applicability of human UFA muscles and MF indices at various force levels of maximum voluntary contraction (MVC). EMG signals are recorded from nine (9) normally limbed subjects. The frequency domain power spectrum density (PSD) is computed in order to derive the useful characteristics of the signal. The results show that only few muscles contributes for the movement. Further analysis show that flexor digitorum superficialis (FDS), flexor carpi radialis (FCR), extensor carpi radialis longus (ECRL), extensor digitorum communis (EDC) and biceps/triceps brachii show interesting results.
\end{abstract}

Keywords: Identification for control; electromyography; muscle fatigue; frequency domain identification; muscle channel estimation.

\section{Introduction}

Many people suffer from losing their forearm or upper arm due to diseases, accidents, or war. In England, there are 44 actively operating prosthetics service centres. Diabetes $42 \%$ and dysvascularity $72 \%$ are the most common reasons for the amputation. There are more than 130 diabetes amputation 
cases reported every week, based on an analysis by Diabetes UK. The annual number of diabetes-related amputations has increased up to 7,000 in 2015. This is $4.83 \%$ of increment from the previous year, 2014. The human UFA amputation statistics in England has increased dramatically with 300 cases per year.

Since the 1980s, there have been numerous studies on the human normal limbed surface EMG signals for movement identification to be used as input for prosthetic arm control. These studies have used a non-invasive recording technique of surface EMG on the forearm ${ }^{1}$ and upper arm. ${ }^{2}$ This shows the importance of such studies in order to help people with such problems to have a better life. The structure of this paper is as follows. Section 2 presents an explanation of EMG, continued with human UFA. Section 3 presents the proposed methodology. Section 4 presents the experimental results, and finally a conclusion is given in Section 5 .

\section{Electromyography}

Electromyography (EMG) is one of the major components in the nerve conduction studies. EMG is one of the techniques for detecting, recording and evaluating the action potential produced by the muscles of the body. It is also known as the diagnostic procedure for the muscle health assessment and the motor neurons control. The origin of EMG action potential or pulse comes from the central nervous system (CNS). ${ }^{3}$ The brain signal is transferred along the nerves through the motor neurons carrying information in pulse repetition or known as frequency. The action potentials generated from this occasion is known as Motor Unit Action Potentials (MUAPs). ${ }^{4,5}$

EMG signals are considered as non-stationary as their characteristics change over time especially on the number and firing rate of motor units, force and joint angle. ${ }^{6}$ Studies have been conducted on the influence of MF on EMG single amplitudes and forces. ${ }^{7}$ They conclude that the amplitudes of EMG are not a viable solution for estimating the muscle activation and the force during fatigue. ${ }^{8} \mathrm{MF}$ is a phenomenon that happens for every creature with muscles in their body such as human and animal. There are many definitions derived from the MF phenomena, and it has been extensively discussed in studies considering the factors involved. One of the best descriptions has been given by ${ }^{9}$ muscles that are used intensively show a progressive decline of performance and basically recovery after a period of rest.

The forearm muscles are divided into four layers, from first to fourth layers, and two compartments (anterior and posterior). Anterior compartment 
is separated by posterior compartment by two bones (ulna and radius), interosseous membrane, and lateral intermuscular septum. ${ }^{10}$ The focus on the current study is on the relations of the forearm muscles and upper arm muscles. The linkages are strong and it is envisaged to develop a good measurement strategy using both regions of the human UFA, to establish suitable means for achieving high classification for dexterous control of prostheses.

The interest is to study and explore the new features with the presence of of such phenomena as MF to help avoid the risk of getting impaired ${ }^{11}$ or probabilities of detecting neuromuscular disorder and disease. GonzalezIzal et. $\mathrm{al}^{12}$ have concluded in thier studies that approaches that associate with several sets of EMG features for measuring force variations yield better understanding about MF. They have also suggested that more study is demanded to expand procedures that combine EMG variables for estimating changes with respect to MF.

\section{Methodology}

To the author's knowledge, there are no studies reported investigating the relations of surface EMG signals of upper arm and forearm muscles region. Accordingly, this research investigates the EMG activity between both muscles and its relation in terms of the signal characteristic. It is believed that the propagation of the EMG signals originates from the brain, towards the muscle fiber from the upper arm and then the forearm back and forth. When the signals propagate from the same source, interconnection between them passing will allow their identification. The relationships must be unique since it is in the same neighborhood or region of interest. The idea is, if a person experiencing an amputation on above elbow, the upper arm muscles can also be used to generate finger or wrist movements and curl exercises. The general scheme of the assessment strategy utilized in this study is the similar to that studied before, ${ }^{13}$ but with different approach where the region of muscles of normally limbed subjects are the main component of the study. Various analytical approaches, such as EMG conditioning and segmentation, feature extraction, dimensionality reduction and classification are considered.

There are nine (9) subjects involved, and aged between 20 and 40 years old. The subjects chosen must be normally limbed without any neuromuscular problems. The subjects are asked about their dominant hand, and will be briefly explained (orally or using visual aid, ie recorded video) and provided with informed consent prior to the study. This study has been 
rewarded an ethical approval by the Ethical Committee of the University of Sheffield, United Kingdom (Department of Automatic Control and Systems Engineering). The muscle selection strategy is completed with EMG signals of hand grip force. The purpose of this stage is to investigate and differentiate which UFA muscles are the most dominant with force variations.

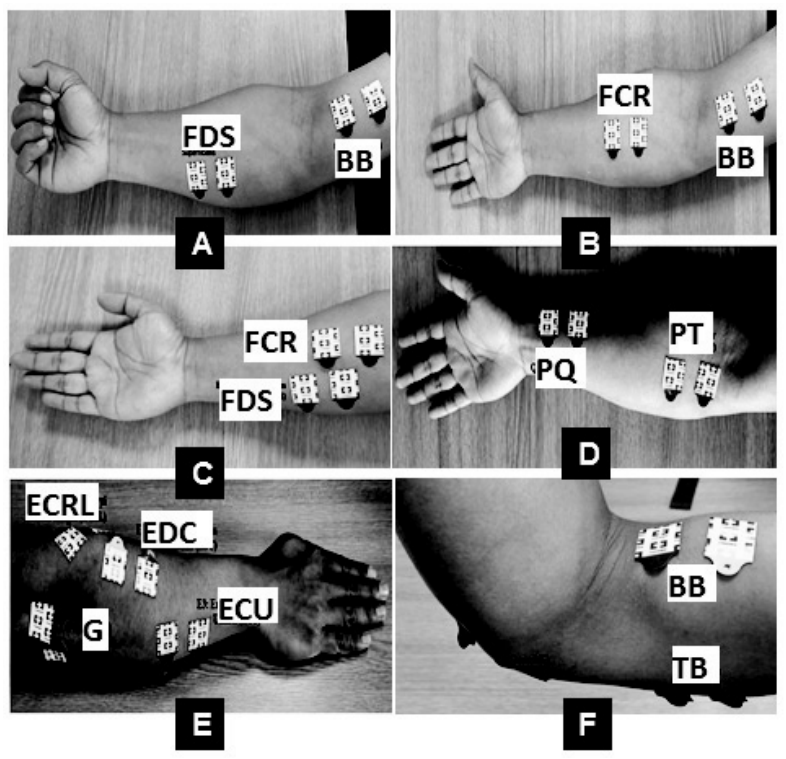

Fig. 1. Example of electrodes placement for the forearm and upper arm muscles as recommended by SENIAM; ${ }^{14}$ (A, B, D and F)Electrode placement for the selected forearm and upper arm muscles involved in pronation and supination with curl exercises, and ( $\mathrm{C}$ and $\mathrm{E})$ Electrode placement for the selected forearm muscles involved in finger(s) pinching and hand grasping.

The study investigates the effect of force variations with MF from normally limbed subject. This will lead to a suitable muscle selection strategy for further analysis. Investigations into the effect of MF with the EMG signal were carried out and five levels of hand grip force with respect to the $(20,40,60,80,100 \% \mathrm{MVC})$ at $90^{\circ}$ angle were recorded. During recording, the subject sat on a chair facing the battery-powered computer with the LabQuest mini interface software. This could help them to visualize the real time EMG signal while performing the movements. The forearm was fixed in one position. At the initial stage, the subject was asked to perform hand grip with maximum force to determine their MVC. This was done 
three times and the average taken as their final MVC. Then, the subject was asked to produce a series of hand grip forces for different percentages of their MVC. This was separated by 5 s rest for each movement. EMG data were collected at sampling frequency of $2000 \mathrm{~Hz}$.

\section{Results}

The MF phenomenon was investigated based on the EMG signal recorded from the subject. However, to achieve fatigue condition, the subject was asked to apply to do maximum hand grip force for a certain amount of time at each level of \%MVC. This can be seen as in Figure 2a. As note the $\%$ MVC was stable at $80 \%$, and then it can be seen that moment reduced as the force increased. This phenomenon is known as muscle fatigue, where the subject struggles to achieve the desired MVC value. As the subject tried to push harder and harder, the force still going down. For all the subjects, $77.7 \%$ of the subjects reached fatigue after $80 \%$ MVC.
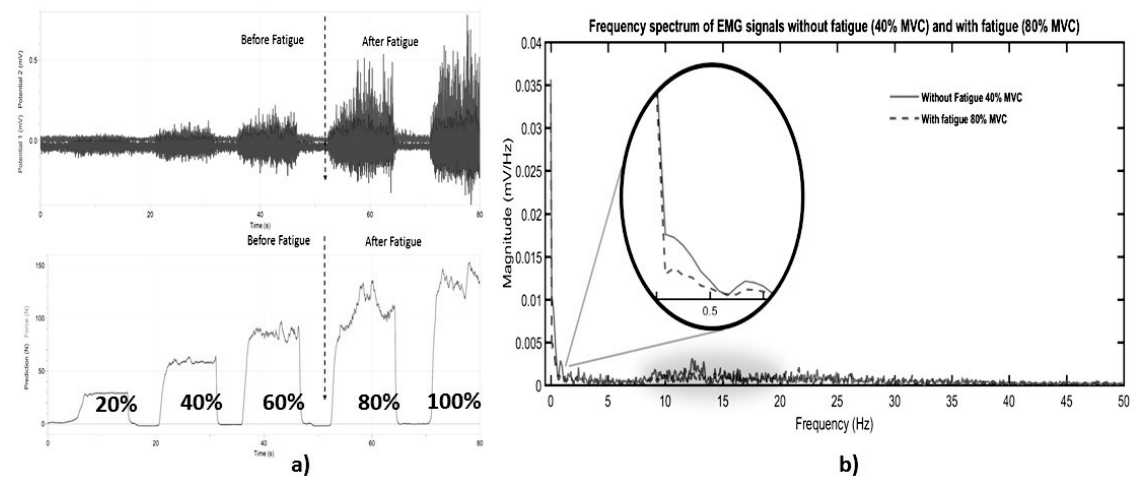

Fig. 2. a) EMG signal of handgrip force with muscle fatigue occurence after $80 \%$ MVC, b) Examples of handgrip EMG frequency spectrum with and without fatigue. The amplitudes is shrinked towards lower frequencies when fatigue occured.

This can be compared with the normal EMG signals, the envelope of this EMG activity, each spike represent the brain activity telling the muscles to be active. More spikes mean more muscle activity, as can be seen the envelope of EMG increased even though the strength was actually decreasing as a function of time. This means that the brain is driving the muscle harder, but something happening peripherally in the muscle makes it weaker than supposed by the brain. This is called MF and the force 
variations degrade the EMG signal performance and cause fatigue. The result of the present study demonstrates that the MF affects the frequency contents of EMG signal. It is shown that the characteristic of the frequency shifts to the lower frequency when the muscle experiences fatigue (Figure 2b. This finding is also supported by previous literatures stated that the amplitude variations shrink towards zero ${ }^{15}$ as the number of MUAPs firing rate increases to maintain the muscle force at increased amplitude. ${ }^{16}$

In this paper, the average powers of muscles involved in human UFA are compared. Examples of power spectrum density (PSD) are illustrated in Figure 2b. The assessment strategy using PSD elucidates the effect of force variations on the EMG signals of human UFA muscles. For human UFA muscles assessment strategy, all the muscle involved were successfully differentiated based on their PSD performances. The illustrated results are the average power of each muscle signal. This will help to understand the behaviour and the performance of individual human UFA muscles. PSD measures of a signal power intensity in the frequency domain. It was computed from the fast fourier transform spectrum of a signal. The amplitude versus frequency data suggest a tendency of few muscles to respond very well with the task performed. This will be a good reference point as these are needed to have suitable muscle selection to reach a plateau in the data consistency and accuracy of the human UFA classification. It was found that there were several muscles from both regions of interest gave good response for the movement. Some of the muscles are producing relatively high inconsistencies such as FCU, FPL, ECU, PT, and PQ.

However, minimising the number of channels is the main focus of the study, five (5) human UFA muscles will be selected. Therefore, FDS, FCR, ECRL and EDC muscles are selected for the forearm, while upper arm muscles show an interesting trend between $\mathrm{TB}$ and $\mathrm{BB}$ and we can use either of its for the future study. It is believed that it could improve the current problems by avoiding the trade-off between adjacent muscles and crosstalk. These are highlighted in the bar chart shown in Figure 3(a). The estimated median and confidence interval of power distribution of muscles involved with force variations shown with the box plot as shown in Figure $3(\mathrm{~b})$. It is shown that the power variance of the human upper forearm muscles are significantly different at the upper boundary of the signal for each muscle. The results shown that only FDS responded well towards force variation of the hand grip where almost all the interested data are within the interquartile range (IQR). The BB and TB muscles still shown different and interesting characteristic among each other. This would be beneficial for 
0.0025

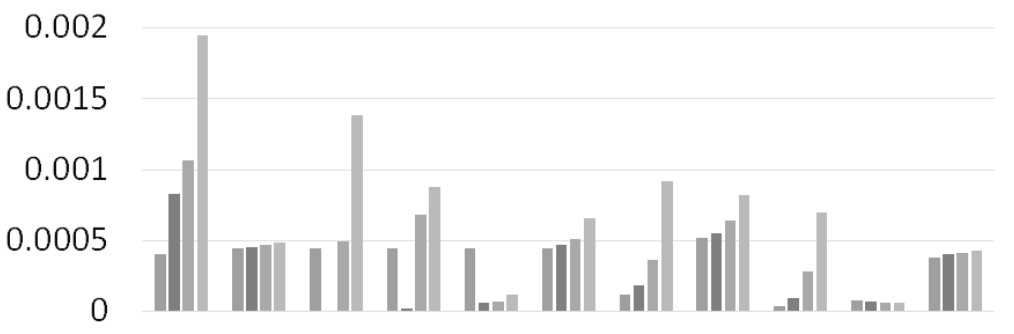

(a) FDS FCR FCU FPL PT PQ ECRL ECU EDC BB TB $\square$ - $20 \% \mathrm{MVC} \quad 40 \% \mathrm{MVC} \square 60 \% \mathrm{MVC} \square 80 \% \mathrm{MVC}$

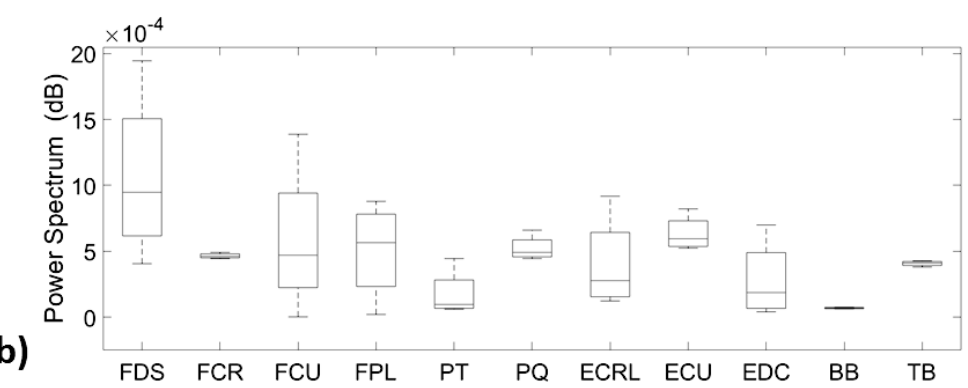

Fig. 3. Human upper forearm muscle performances; (a) bar chart for average power estimation produced by muscles with respond to the force variations, (b) box-plot of estimated median and confidence interval for each muscle.

developing new features involving two regions of muscles for classification.

\section{Conclusion}

This study explored and evaluated the new approaches of data collection and assessing the human upper forearm muscles with force variations, as well as muscle fatigue. It is suggested that the most applicable use of muscle is to establish the inter-relation between two regions of human upper forearm. Although experimental protocols were implemented for the hand grip movement, results show that this could achieve the objective of study. The assessments and conclusions are not definitive solutions; however, they contribute to some extent to advance knowledge in the field. This study offers an opportunity to develop new feature and classification scheme to enhance the capabilities of disable people especially those that may have problems of weaknesses in the muscle contraction or are amputated. The current study focuses on paralysed persons or those amputated at the 
middle region between the forearm and upper arm, where the current single system approach will not be adequate.

\section{Acknowledgment}

W. M. B Wan Daud and N. Abas are on study leave and sponsored by Universiti Teknikal Malaysia Melaka (UTeM) and Ministry of Higher Education of Malaysia.

\section{References}

1. P. C. Doerschuk, D. E. Gustafon and A. S. Willsky, IEEE Transactions on Biomedical Engineering BME-30, 18 (1983).

2. J. M. Winters and D. G. Kleweno, Journal of Biomechanics 26, 143 (1993).

3. K. Anam, R. N. Khushaba and A. Al-Jumaily, Two-channel surface electromyography for individual and combined finger movements, in 2013 35th Annual International Conference of the IEEE Engineering in Medicine and Biology Society (EMBC), 2013.

4. R. B. Stein and H. S. Milner-Brown, Contractile and Electrical Properties of Normal and Modified Human Motor Units, in Control of Posture and Locomotion, eds. R. B. Stein, K. G. Pearson, R. S. Smith and J. B. Redford (Springer US, Boston, MA, 1973), Boston, MA, pp. 73-86.

5. L. J. Hargrove, K. Englehart and B. Hudgins, IEEE Transactions on Biomedical Engineering 54, 847 (2007).

6. A. D. Cechetto, P. A. Parker and R. N. Scott, Journal of Electromyography and Kinesiology 11, 347 (2001).

7. J. L. Dideriksen, D. Farina and R. M. Enoka, Philosophical Transactions of the Royal Society of London A: Mathematical, Physical and Engineering Sciences 368, 2765 (2010).

8. R. N. Khushaba and S. Kodagoda, Electromyogram (emg) feature reduction using mutual components analysis for multifunction prosthetic fingers control, in Control Automation Robotics and Vision (ICARCV), 2012 12th International Conference on, 2012.

9. D. G. Allen, G. D. Lamb and H. Westerblad, Physiological Reviews 88, 287 (2008).

10. S. H. Parson, Journal of Anatomy 215, 474 (2009).

11. F. Monjo, Human Movement Science 44, 225 (2015).

12. M. Gonzlez-Izal, A. Malanda, E. Gorostiaga and M. Izquierdo, Journal of Electromyography and Kinesiology 22, 501 (2012).

13. A. H. Al-Timemy, G. Bugmann, J. Escudero and N. Outram, Biomedical and Health Informatics, IEEE Journal of 17, 608 (2013).

14. R. Merletti, Europa Medicophysica 36, 167 (2000).

15. G. Venugopal, M. Navaneethakrishna and S. Ramakrishnan, Expert Systems with Applications 41, 2652 (2014).

16. R. M. Enoka, S. Baudry, T. Rudroff, D. Farina, M. Klass and J. Duchateau, Journal of Electromyography and Kinesiology 21, 208 (2011). 